\title{
Hybrid-lens for Terahertz Subwavelength Focusing beyond the Diffraction Limit
}

\author{
Piyawath Tapsanit ${ }^{1,2, a}$, Masatsugu Yamashita ${ }^{2, \mathrm{~b}, *}$, and Chiko Otani ${ }^{1,2}$ \\ 1 Department of Physics, Tohoku University, Sendai 980-8578, Japan \\ 2 RIKEN Center for Advanced Photonics, Aramaki-Aoba, Aoba-ku, Sendai 980-0845, Japan \\ E-mail: apiyawath.tapsanit@riken.jp, ${ }^{\mathrm{b} m-y a m a @ r i k e n . j p ~(C o r r e s p o n d i n g ~ a u t h o r) ~}$
}

\begin{abstract}
The relatively low energy of the terahertz light $(\mathrm{THz}, 1 \mathrm{THz}=4.14 \mathrm{meV}$ ) promises the non-invasive optical imaging of the biomedical objects. However, the $\mathrm{THz}$ light inherently suffers the optical diffraction limit and the subwavelength microstructures cannot be resolved. We propose the planar hybrid-lens comprising the multiple slits and the two-dimensional rod array for the subwavelength focusing of the $\mathrm{THz}$ light beyond the diffraction limit. The hybrid-lens is investigated by the finite difference time domain simulation (FDTD). The spectra of the rod array obtained by FDTD are compared with the spectra calculated by the analytical model. The subwavelength focal spot about $20 \mu \mathrm{m}$ $(\lambda / 7.5)$ at the frequency $2 \mathrm{THz}$ is demonstrated by FDTD. The array of the subwavelength focal spots on the opposite side of the multiple slits are generated by the interferences of the Floquet-Bloch waves which can be guided through the rod array. The hybrid-lens can function as the subwavelength focusing and the substrate simultaneously which may serve as the new tool in the high-speed imaging and sensing of the subwavelength biomedical objects by using the $\mathrm{THz}$ light.
\end{abstract}

Keywords: Terahertz, FDTD, diffraction limit, subwavelength focusing, multiple slits, rod array.

ENGINEERING JOURNAL Volume 20 Issue 1

Received 28 February 2015

Accepted 26 June 2015

Published 29 January 2016

Online at http://www.engj.org/

DOI:10.4186/ej.2016.20.1.61 


\section{Introduction}

The optical imaging using the $\mathrm{THz}$ light is expected to be new medical imaging system because of its nondestructive nature and its relatively low energy $[1,2,3]$. However, the optical diffraction limit severely restricts the focusing spot of light and blur the optical image. The diffraction limit is the inherent consequence of the circular dispersion relation of the isotropic medium which does not support the propagation of the evanescent waves carrying the high spatial frequencies [4]. The strongly anisotropic medium such as the hyperlens is the material exhibiting the hyperbolic dispersion relation with the unbound spatial frequency thereby allowing the propagation of the evanescent waves with the high spatial frequencies (high-k waves) $[5,6,7,8]$. Unfortunately, the hyperlens is hardly found in nature, and it is conventionally engineered using the idea of the photonic crystals (Phc) and metamaterials into many shapes, e.g., cylindrical hyperlens, planar hyperlens, and fibre hyperlens [7, 9, 10]. Recently, A. Tuniz et al. have experimentally demonstrated the $\mathrm{THz}$ tapered-fibre hyperlens working in the canalisation regime [10]. The size of the focal spot of this lens depends on the diameters of the input and output sides of the taper. The subwavelength focal spot with size about $143 \mu \mathrm{m}(\lambda / 28)$ at the low frequency $\mathrm{f}=0.075 \mathrm{THz}$ is demonstrated. However, the aberrations deteriorate the focusing at higher frequencies due to the intrinsic loss and the large local dielectric constant along the propagation direction of the hyperlens [10,11], and therefore the smaller focal spot size can not be achieved by using this lens.

In this research, the planar hybrid-lens supporting the propagation of the $\mathrm{THz}$ waves with high- $\mathrm{k}$ similar to the hyperlens is proposed by combining the multiple slits with the array of the metallic rods embedded in the polymer host. The rod array is closely separated along the normal axis allowing the strong coupling of the surface charges between the rods, and the period of the rod array along the tangential axis is wider than that of the normal axis. The multiple slits generate the Floquet-Bloch waves which are then guided through the rod array, interfere with each other, and become focused on the opposite side of the multiple slits. The array of the subwavelength focal spots can be employed in the non-invasive and fast $\mathrm{THz}$ imaging and sensing of the subwavelength objects. Moreover, the focal spot size may be further reduced by optimizing the parameters of the multiple slits and the rod array. Therefore, our hybrid-lens also gives the alternative approach for realising the deep-subwavelength focusing of the $\mathrm{THz}$ light. The proposed hybrid-lens can be readily fabricated by using the available methods [12].

\section{Method}

The two-dimensional schematic view of the unit cell of the planar hybrid-lens and the rectangular unit cell of the rod array are shown in Figs. 1(a)-(b), respectively. The metallic rods and the multiple slits are modeled as the perfect electrical conductors (PEC) which are valid for noble metals such as gold, silver and aluminium in terahertz and microwave spectral regions [13]. This approximation makes no loss in the structure, reduces the memory usage in the FDTD simulation, and enhances the calculation time. The polymer host is low loss Zeonex polymer [14].

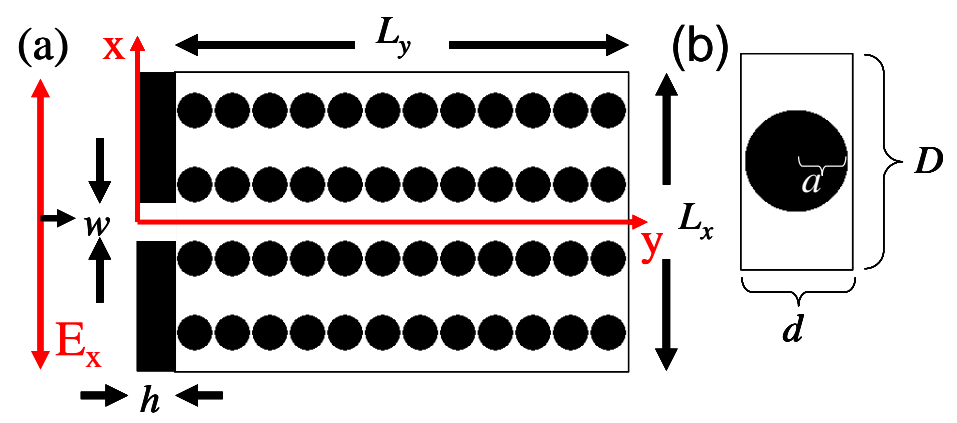

Fig. 1. The schematic view of the hybrid-lens: (a) The unit cell of the hybrid-lens comprising the multiple slits and the two-dimensional rod array; (b) The rectangular unit cell of the rod array. The multiple slits and the metallic rods are modeled as the PEC rods, and the dielectric host is defined as low loss Zeonex. 
The parameters of the rod array are fixed and used throughout the paper as $D=20 \mu \mathrm{m}, \mathrm{d}=10 \mu \mathrm{m}$, and $\mathrm{a}=4.8 \mu \mathrm{m}$. However, the length $\mathrm{L}_{\mathrm{x}}$ and $\mathrm{L}_{\mathrm{y}}$ in Fig. 1(a) may be varied in the simulation. The slit height (h) and the slit width (w) are defined as $\mathrm{h}=1 \mu \mathrm{m}$, and $\mathrm{w}=20 \mu \mathrm{m}$, respectively. The slit cavity is filled with air and thus behaves as the waveguide. The slit height (h) is small comparing to the wavelength of light $(\lambda)$ so that the Fabry-Perot resonances do not occur inside the slit cavity [15]. The slit width is several time larger than the typical skin depth, the distance at which the electric field decays by $1 / e$ inside the slab, of the noble metals and thus PEC approximation is applicable to the multiple slits (Au : $176.1 \mathrm{~nm}$ at $0.5 \mathrm{THz}, 67.0$ at 3.0 $\mathrm{THz}$ ). The full Maxwell's equations are solved numerically by using FDTD method. We employ the freelydistributed parallel FDTD program called MEEP in our research [16].

It is difficult to derive the spectra of the hybrid-lens analytically because the coupling between the slit's waveguide modes and the modes of the rod array must be taken into account [15]. Here, we only show the spectra of the rod array (not including the multiple slits) obtained analytically by modeling each metallic rod as the electric dipole moment, regarding the single layer of the metallic rods which is perpendicular to the $y-$ axis as the homogeneous medium possessing the unique reflection and transmission coefficients $r_{0}$ and $t_{0}$, respectively, and then applying the transfer matrix method for the whole $N$ layers of rod array, as described by G. D'Aguanno et al. [17]. The analytical 0th-order reflectance and transmittance of the $N$ layers of rod array (e.g., $N=12$ in Fig. 1(a)) for the $p$-polarized light are expressed as

$$
R=\left|S_{21} / S_{11}\right|^{2}, T=1 /\left|S_{11}\right|^{2},
$$

where $S_{i j}$ is the matrix element of the scattering matrix $[S]$ which is defined as

$$
[S]=\left[M_{\text {in }}\right][V]^{N-2}\left[M_{\text {out }}\right],
$$

where the matrices $\left[M_{\text {in }}\right],[V]$, and $\left[M_{\text {out }}\right]$ are expressed in terms of $r_{0}$ and $t_{0}$ as

$$
\begin{aligned}
& {\left[M_{\mathrm{in}}\right]=\frac{e^{i k_{y}^{\mathrm{in}} d / 2}}{t_{\mathrm{in}, \mathrm{b}} t_{0}}\left[\begin{array}{ll}
r_{\mathrm{in}, \mathrm{b}} r_{0}+e^{-i k_{y}^{\mathrm{in}} d} & r_{\mathrm{in}, \mathrm{b}}\left(t_{0}^{2}-r_{0}^{2}\right) e^{i k_{y}^{\mathrm{in} d}}-r_{0} \\
r_{0}+r_{\mathrm{in}, \mathrm{b}} e^{-i k_{y}^{\mathrm{in}} d} & \left(t_{0}^{2}-r_{0}^{2}\right) e^{i k_{y}^{\mathrm{in}} d}-r_{\mathrm{in}, \mathrm{b}} r
\end{array}\right],} \\
& {[V]=\frac{1}{t_{0}}\left[\begin{array}{cc}
e^{-i k_{y}^{\text {in } d}} & -r_{0} \\
r_{0} & \left(t_{0}^{2}-r_{0}^{2}\right) e^{i k_{y}^{\text {in }} d}
\end{array}\right],} \\
& {\left[M_{\text {out }}\right]=\frac{e^{-i k_{y}^{\text {in } d}}}{t_{\mathrm{b}, \text { out }} t_{0}}\left[\begin{array}{cc}
-\left(r_{\mathrm{b}, \text { out }} r_{0}-e^{-i k_{y}^{\text {ind }}}\right) & -t_{\mathrm{b}, \text { out }} r_{0} \\
r_{0}+r_{\mathrm{b}, \text { out }}\left(t_{0}^{2}-r_{0}^{2}\right) e^{i k_{y}^{\text {ind } d}} & t_{\mathrm{b}, \text { out }}\left(t_{0}^{2}-r_{0}^{2}\right) e^{i k_{y}^{\text {ind }} d}
\end{array}\right],}
\end{aligned}
$$

and

where $k_{y}^{\text {in }}$ is the normal component of the incident wavevector, $t_{\mathrm{a}, \mathrm{b}}$ and $r_{\mathrm{a}, \mathrm{b}}$ are the Fresnel transmission and reflection coefficients, respectively, from the medium (a) to the medium (b) [18]. The reflection and transmission coefficient, $r_{0}$ and $t_{0}$, of the single layer of the rod array generated by the re-radiation of the electric dipole moments $\vec{p}=\alpha \vec{E}_{\text {in }}=p_{x} \hat{x}+p_{y} \hat{y}$ of all metallic rods in that layer are obtained by the Green's function analysis $[17,18]$ which then are written as

$$
r_{0}=\frac{i}{2 D}\left(\frac{\omega p_{x}}{H_{0}}+\frac{k_{x}^{\text {in }}}{k_{y}^{\text {in }}} \frac{\omega p_{y}}{H_{0}}\right), t_{0}=1-\frac{i}{2 D}\left(\frac{\omega p_{x}}{H_{0}}-\frac{k_{x}^{\text {in }}}{k_{y}^{\text {in }}} \frac{\omega p_{y}}{H_{0}}\right),
$$

where $k_{x}^{\text {in }}, \omega$, and $H_{0}$ are the tangential component of the incident wavevector, the angular frequency, and the amplitude of the incident magnetic field, respectively. The electric dipole moment $\vec{p}$ is periodic along the x-axis with the period $D$. By following [19], the components of $\vec{p}$ can be expressed in terms of the electric polarizability $\alpha$ of the metallic rod as

$$
p_{x}=\frac{-k_{y}^{\text {in }}}{\varepsilon_{0} \varepsilon_{b} / \alpha-\left(k_{b}^{2} G_{b}+\partial_{x}^{2} G_{b}\right)}, p_{y}=\frac{k_{x}^{\text {in }}}{\varepsilon_{0} \varepsilon_{b} / \alpha-\left(k_{b}^{2} G_{b}+\partial_{y}^{2} G_{b}\right)},
$$

where $G_{b}, \partial_{x}^{2} G_{b}$, and $\partial_{y}^{2} G_{b}$ are given in [20], and $k_{b}$ is the wavenumber in the host medium of the rod array. Lastly, the electric polarizability $\alpha$ can be obtained by matching the electromagnetic waves radiated 
by the electric dipole with the cylindrical waves scattered from the single cylindrical rod which is excited by the external light [21], and then $\alpha$ is expressed in this unit as

$$
\alpha=8 i \varepsilon_{0} \varepsilon_{b} \frac{1}{k_{b}^{2}}\left\{\frac{\sqrt{\varepsilon_{\mathrm{r}} / \varepsilon_{\mathrm{b}}} J_{1}^{\prime}\left(k_{\mathrm{b}} a\right) J_{1}\left(k_{\mathrm{r}} a\right)-J_{1}\left(k_{\mathrm{b}} a\right) J_{1}^{\prime}\left(k_{\mathrm{r}} a\right)}{\sqrt{\varepsilon_{\mathrm{r}} / \varepsilon_{\mathrm{b}}} H_{1}^{(1) \prime}\left(k_{\mathrm{b}} a\right) J_{1}\left(k_{\mathrm{r}} a\right)-H_{1}^{(1)}\left(k_{\mathrm{b}} a\right) J_{1}^{\prime}\left(k_{\mathrm{r}} a\right)}\right\},
$$

where $J_{1}$ is the first order Bessel function, $H_{1}^{(1)}$ is the first order Hankel function of the first kind, $\varepsilon_{\mathrm{r}}$ and $k_{\mathrm{r}}$ are the dielectric constant and the wavenumber of the rod, and the apostrophe means the derivative with respect to the argument. The dielectric constant of gold is used when we apply the analytical solutions.

\section{Results}

We show the spectra of the rod array in section 3.1 and discuss the result with the analytical theory. Then, the field distribution at the frequency $2 \mathrm{THz}$ is shown in section 3.2. Lastly, the subwavelength focusing at the same frequency is demonstrated in section 3.3.

\subsection{The Spectra of the Rod Array for $\mathbf{E}_{\mathrm{x}}$ and $\mathbf{E}_{\mathrm{y}}$ Polarizations}

The reflection and transmission spectra of the rod array in the frequency range of interest for the $E_{y}$ incident light obtained by FDTD simulation with the resolution 4 pixels/ $\mu \mathrm{m}$ are shown in Fig. 2(a). The length $L_{x}$ is finite and defined as $L_{x}=4 D=80 \mu \mathrm{m}$, while the periodic boundary condition is applied along the $\mathrm{y}$-axis. The period of the single layer of the rod array along the $\mathrm{y}$-axis is equal to $\mathrm{d}=10 \mu \mathrm{m}$. The spectra saturates by increasing the resolution and become converged at about 4 pixels $/ \mu \mathrm{m}$. The resolution of 4 pixels $/ \mu \mathrm{m}$ means that the space discretization is $\Delta \mathrm{x}=0.25 \mu \mathrm{m}$ (the same in two directions of the computational cell) and the time discretization is $\Delta \mathrm{t}=0.5 \Delta \mathrm{x} / \mathrm{c}=416.955 \mathrm{ps}$, where $\mathrm{c}$ is the speed of light. The rod array is excited by the normal incidence of the Guassian pulse generated at the far-field plane, and the perfectly matched layers (PMLs) are applied on two boundaries of the computational cell which are perpendicular to the propagation direction in order to prevent the back-scattering. The reflectance and transmittance are obtained by the ratio between the power of the scattering light from the rod array and the power of the incident light which are measured in the far-field region in front of the rod array and behind the rod array, respectively. According to Fig. 2(a), the spectra show the Bragg gap with the edge at the lower energy side about $1.75 \mathrm{THz}$. The electromagnetic waves in this Bragg gap are tightly localized between the rods along the $y$-axis due to the strong coupling of the electric dipoles induced on each rod. The localization of the electromagnetic waves is required for the subwavelength focusing. In the frequency range below $1.75 \mathrm{THz}$, the insulator-like interference peaks appear, and thus there is no strong coupling of the electric dipoles of the metallic rods. We compare the reflection spectra obtained by FDTD from Fig. 2(a) with the analytical solution calculated by Eq. (1) in Fig. 2(b). The analytical reflection spectra are qualitatively consistent with the FDTD reflection spectra. Indeed, the edge at the lower energy is shifted to the higher frequency. The discrepancy may arise because of the inefficiency of FDTD which apply the grid cells on the circular curves of the metallic rods. Therefore, this result reminds us that the results from FDTD are only the approximation giving us the rough pictures of the spectra, and one should not entirely rely on it. Another approach should be considered in order the check the results from FDTD.

The reflection and transmission spectra of the rod array for the $E_{x}$ incident light obtained by FDTD simulation are shown in Fig. 3(a). The length $\mathrm{L}_{\mathrm{y}}$ is finite and defined as $\mathrm{L}_{\mathrm{y}}=12 \mathrm{~d}=120 \mu \mathrm{m}$, while the periodic boundary condition is applied along the $\mathrm{x}$-axis. The remaining parameters of the FDTD simulation are the same as the previous case. The period of the single layer of the rod array along the $\mathrm{x}$-axis in this case becomes $\mathrm{D}=20 \mu \mathrm{m}$ which is therefore two times larger than the previous case. Figure 3(a) shows that the rod array behaves like an insulator for the $\mathrm{E}_{\mathrm{x}}$ polarization. This behavior results from the blue shift of the edge at the lower energy side of the Bragg gap to the higher frequency by increasing the period of the single layer of the rod array. Figure 3(b) shows the comparison of the transmission spectra obtained by FDTD and from the analytical solution expressed in Eq. (1). The qualitative agreement between the FDTD and the analytical solution for the $E_{x}$ polarization can be seen in this figure. 

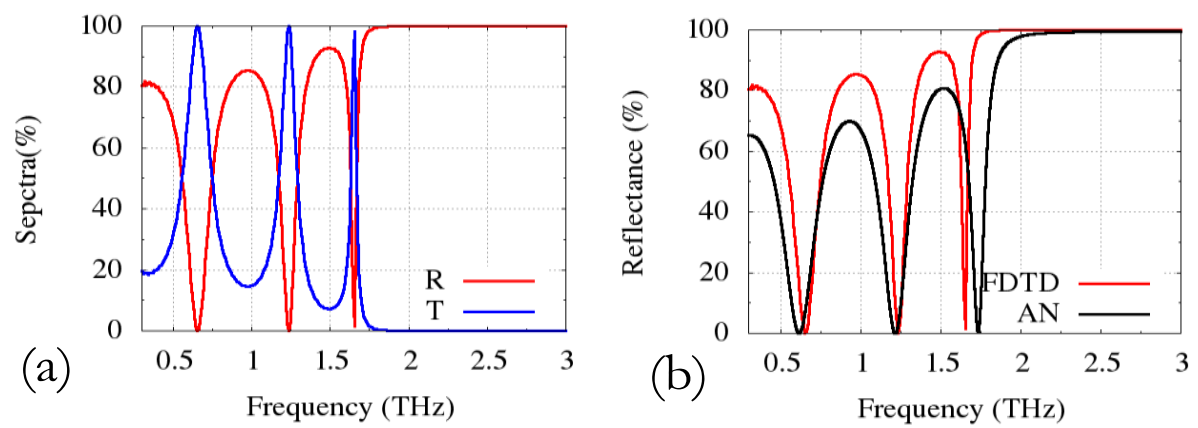

Fig. 2. The spectra for the $\mathrm{E}_{\mathrm{y}}$ polarization (a) the reflection and transmission spectra obtained by FDTD with the convergent resolution 4 pixels/ $\mu \mathrm{m}$, (b) The comparison of the reflection spectra obtained by FDTD and the analytical solution expressed in Eq. (1). The length of the rod array along the yaxis is $\mathrm{L}_{\mathrm{x}}=4 \mathrm{D}=80 \mu \mathrm{m}$.

Therefore, the strongly anisotropic at the frequency $2 \mathrm{THz}$ is expected according to Fig. 2(a) and 3(a). Although we could not determine accurately the effective dielectric constant corresponding to each polarization in order to decide the location of the multiple slits, we still can use the FDTD simulation to investigate the light propagation inside the rod array. Then, we can choose the position of the multiple slits based on the distribution of the electromagnetic fields inside the rod array. We'll describe the distribution of the electromagnetic waves in the next section.
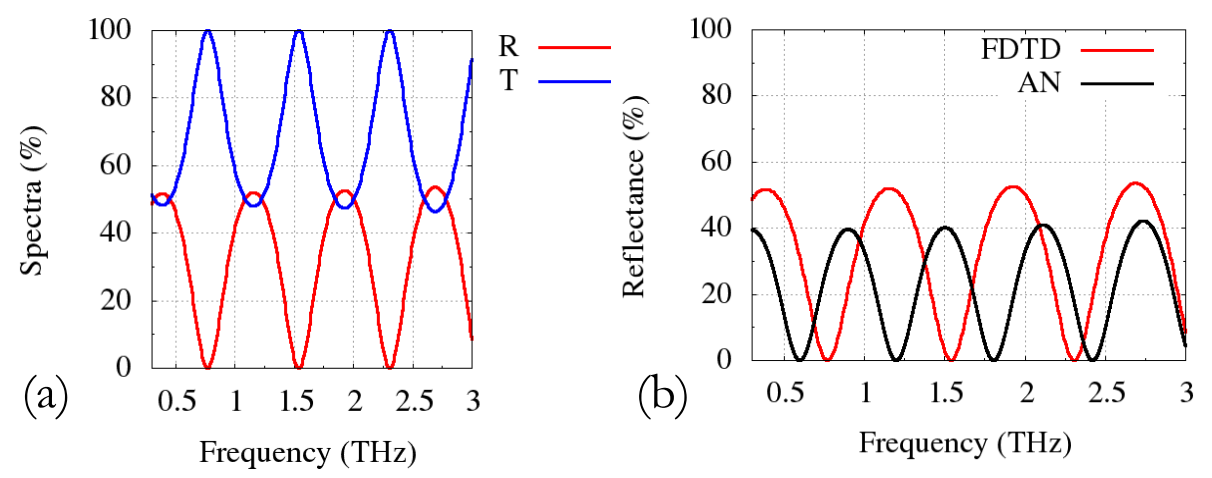

Fig. 3. The spectra for the $E_{x}$ polarization (a) the reflection and transmission spectra obtained by FDTD with the convergent resolution 4 pixels/ $\mu \mathrm{m}$, (b) The comparison of the reflection spectra obtained by FDTD and the analytical solution expressed in Eq. (1). The length of the rod array along the yaxis is $\mathrm{L}_{\mathrm{y}}=12 \mathrm{~d}=120 \mu \mathrm{m}$.

\subsection{Field Distribution Inside the Rod Array}

For the hyperlens with known dielectric constants $\varepsilon_{x}>0, \varepsilon_{y}<0$, the high-k waves propagate in definite direction with respect to the y-axis with the critical angle $\alpha_{c}=\tan ^{-1}\left(\sqrt{\left|\varepsilon_{y}\right| / \varepsilon_{x}}\right)$. Using the facts that $\vec{D} \perp \vec{k}$ and $\vec{S} \perp \vec{E}$, the critical angle of the Poynting vector $\vec{S}$ with respect to the y-axis can also be expressed explicitly in terms of the dielectric constants : $\theta_{c}=\tan ^{-1}\left(\sqrt{\varepsilon_{x} /\left|\varepsilon_{y}\right|}\right)$ [9]. However, we cannot obtain the effective dielectric constants of the hybrid-lens at the present, so we'll check the directional propagation of the high-k waves inside the rod array by exciting it with the point source instead. 


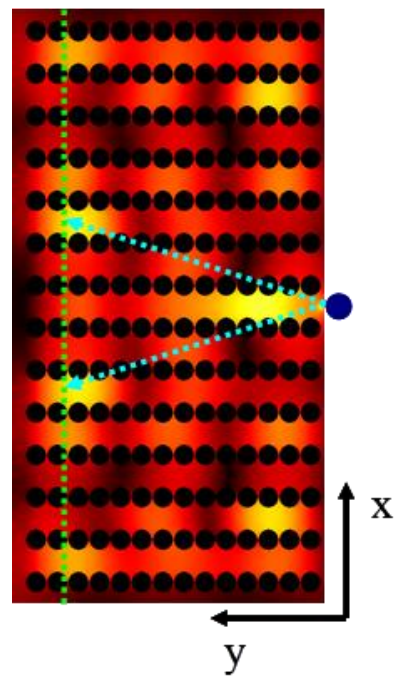

Fig. 4. The field distribution inside the rod array.

The high-k waves are generated by the magnetic current point source $H_{z}$ with the frequency $2 \mathrm{THz}$ and located at one interface of the rod array. Because the intensity is obtained by the amplitude of the Poynting vector which is proportional to $\left|H_{z}\right|^{2}$, we plot $\left|H_{z}\right|$ in Fig. 4 to show the directional property of the intensity inside the rod array. The length of the rod array is $\mathrm{L}_{y}=14 \mathrm{~d}, \mathrm{~L}_{\mathrm{x}}=14 \mathrm{D}$, and the PMLs are defined on boundaries of the computational cell to avoid the back-scattering. According to Fig. 4, $\tan \left(\theta_{c}\right)=2 D / 12 d=1 / 3$, and thus $\theta_{c}$ is about 18.4 degree. Therefore, if two slits which act as the point sources are kept at the separation distance 4D or $80 \mu \mathrm{m}$ from each other, the high-k waves will be focused between these two slits on the plane located at the distance $12 \mathrm{~d}$ or $120 \mu \mathrm{m}$ from the slits. This is our main strategy to design the sub-wavelength focusing hybrid-lens using FDTD simulation. We cannot obtain the effective dielectric constant of the rod array because the fitting effective dielectric constants for each polarization depend on the thickness of the rod array. It is also noted here that the directional propagation can be altered by combining the rod array with the multiple slits because of the coupling between the slit waveguide modes and the electromagnetic waves in the rod array.

\subsection{Subwavelength Focusing of the Hybrid-Lens}

We demonstrate the sub-wavelength focusing using our proposed planar hybrid-lens schematically shown in Fig. 1 at the frequency $2 \mathrm{THz}$ in this section. The length $\mathrm{L}_{\mathrm{x}}$ and $\mathrm{L}_{\mathrm{y}}$ of the unit cell of the hybrid-lens are fixed as $L_{x}=4 D$, and $L_{y}=12 \mathrm{~d}$, respectively, so that the focal spots between the slits are generated along the interface of the rod array in the opposite side of the multiple slits. Figure 5(a) shows the intensity distribution calculated by the amplitude of the Poynting vector over the xy-plane. The normalized intensity profiles along the lines parallel to the x-axis behind the hybrid-lens are shown in Fig. 5(b). The focal spot size is determined by the full-width-at-half-maximum (FWHM) of the main peak power in the intensity profile. At the interface $(0 \mu \mathrm{m})$, the focal spot size is about $20 \mu \mathrm{m}$ or $\mathrm{D}$, and the intensity of the peak exceed unity. The focal spot becomes broad as the distance from the interface increase and the intensity becomes almost flat at the distance about $20 \mu \mathrm{m}$ from the interface. The intensity of the focusing spot decays exponentially as a function of normal distance $(\Delta y)$ from the interface with the decay length, $\Delta y$, at which the intensity decays by $1 / e$, is about $16 \mu \mathrm{m}$ as shown in Fig. 6(a). Therefore, the hybrid-lens is suitable for thin film sensing and imaging but has poor performance for bulk material. The intensity distribution shown in Fig. 5(a) looks very much like the directional propagation inside the hyperlens where the high-k waves are launched from the slits and becomes focused at the specific locations behind the hyperlens [9]. 

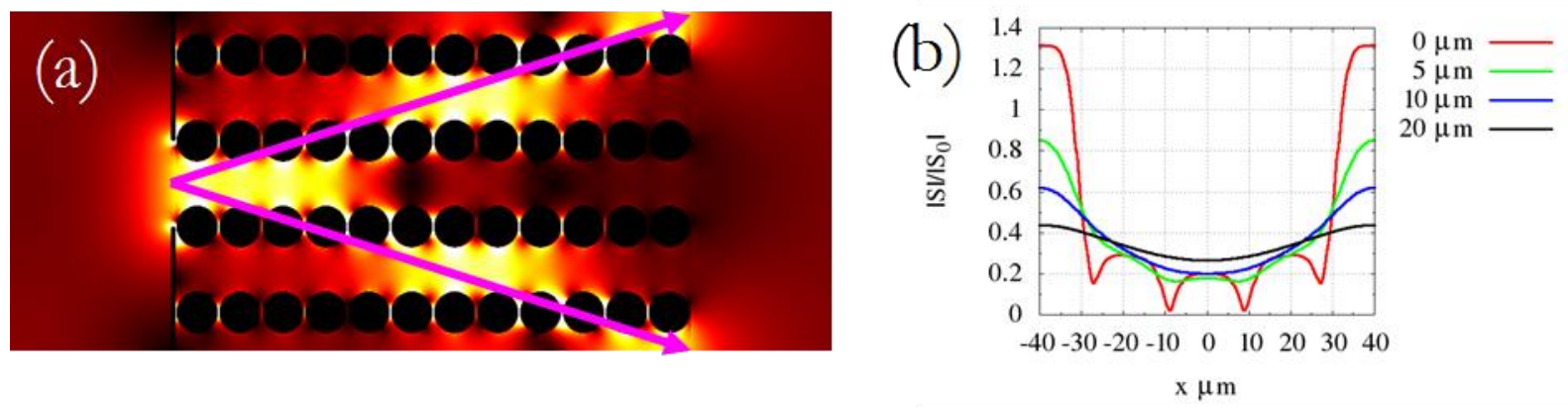

Fig. 5. Subwavelength focusing at $2 \mathrm{THz}$ (a) The intensity distribution calculated by the amplitude of the Poynting vector. The lengths $\mathrm{L}_{\mathrm{y}}=12 \mathrm{~d}$ and $\mathrm{L}_{\mathrm{x}}=4 \mathrm{D}$ of the unit cell yield the constructive interferences of the evanescent waves between each slit pair. (b) The normalized intensity profiles along the lines parallel to the the $\mathrm{x}$-axis at some distances behind the hybrid-lens. The FWHM of the red line is about $20 \mu \mathrm{m}$ or $\mathrm{D}$.
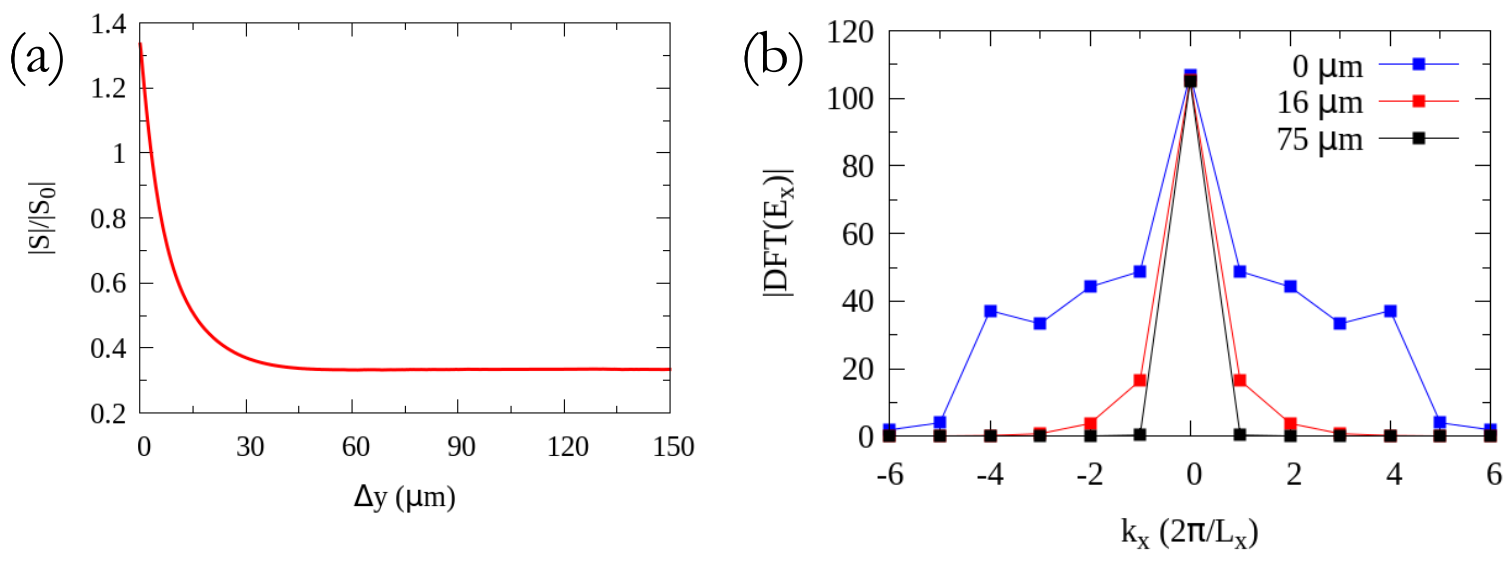

Fig. 6. (a) The intensity as a function of normal distance from the interface of the hybrid-lens at $x=\mathrm{L}_{\mathrm{x}} / 2$. (b) Discrete Fourier Transform (DFT) of $E_{x}$ along the lines parallel to the interface of the hybridlens at three normal distances: (i) $\Delta y=0 \mu \mathrm{m}$ at the focal plane, (ii) $\Delta y=16 \mu \mathrm{m}$ at the decay length, and (iii) $\Delta y=75 \mu \mathrm{m}=\lambda / 2$ at the far-field limit.

In order to understand the focusing mechanism, we perform the Discrete Fourier Transform (DFT) of the tangential component of the electric field $\left(\mathrm{E}_{\mathrm{x}}\right)$ along the lines parallel to the interface between the hybrid-lens and the output region which is defined as the vacuum (red line in Fig. 5(b)). The corresponding DFT of $E_{x}$ at this frequency are shown in Fig. 6(b) at three normal distances from the interface. Note that $2 \pi / \mathrm{L}_{\mathrm{x}}$ is equivalent to $1.874 \mathrm{k}_{0}$, where $\mathrm{k}_{0}$ is the wavenumber in the vacuum. It can be seen from Fig. $6(\mathrm{~b})$ that the high-k waves exist along the focal plane $(\Delta y=0)$ and completely evanescent in the far-field limit $(\Delta y=75 \mu \mathrm{m}=\lambda / 2)$. These high- $\mathrm{k}$ waves are actually the Floquet-Bloch waves (FB) which are generated by the multiple slits. They are just the results of the periodicity with the period $\mathrm{L}_{\mathrm{x}}$ of the multiple slits along the x-axis which force them to satisfy the condition: $H_{z}\left(x+n L_{x}, \mathrm{y}\right)=e^{i k_{x}^{\text {in }} n L_{x}} H_{z}(x, \mathrm{y})$, where $n$ is an integer [22]. The tangential components of the wavevectors of these waves can be as large as $k_{x}=4\left(2 \pi / L_{x}\right)$. If there was no rod array behind the multiple slits, these waves would exponentially decay and didn't exist along this plane. Interestingly, by adding the rod array, these high-k waves can propagate through the rod array and become focused along this plane. Therefore, the hybrid-lens comprising the multiple slits and the rod array serves as the metamaterial supporting the propagation of the FB waves with high-k. The subwavelength focal spots along the red line in Fig. 5(b) are generated by the interferences of these FB waves with $k_{\mathrm{x}}$ up to $4\left(2 \pi / \mathrm{L}_{\mathrm{x}}\right)$. The FB waves also make internal reflection along the focal plane because of the mismatch of the wavevectors between the hybrid-lens and the output region. Therefore, the 

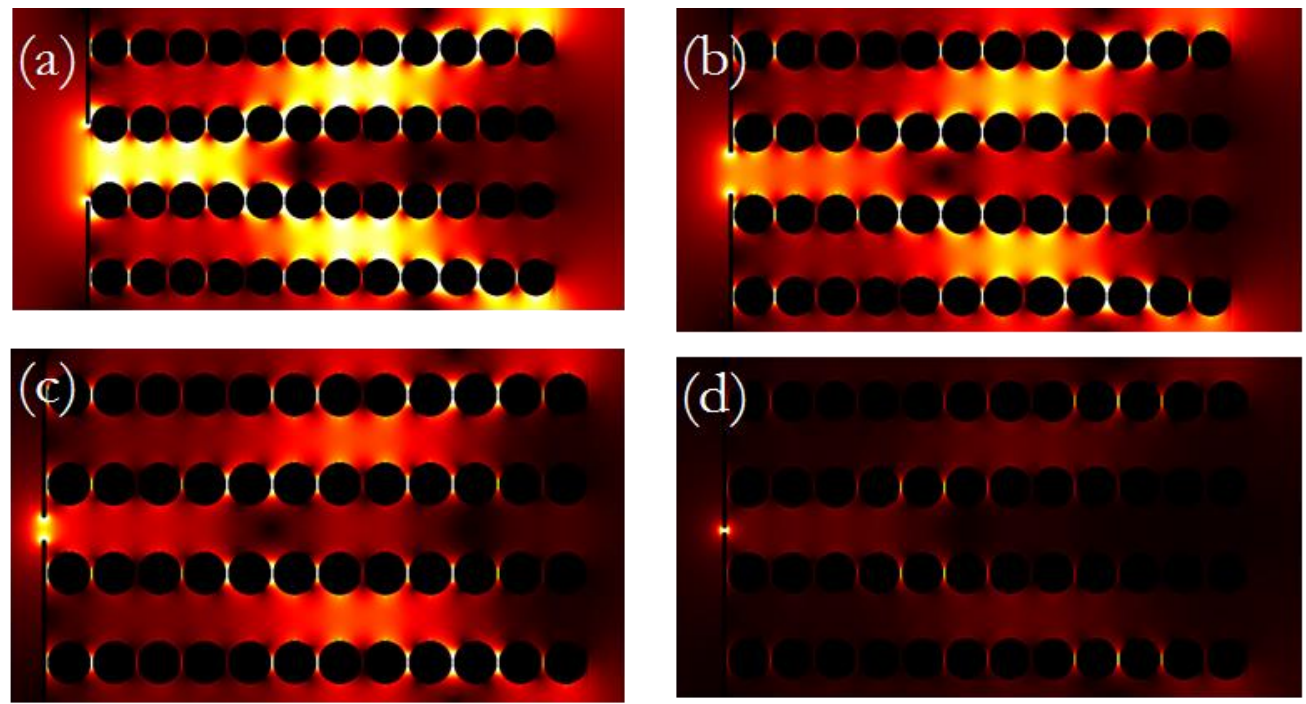

Fig. 7 Effect of the slit width on the subwavelength focusing at 2 THz: (a) w=20 $\mu \mathrm{m},(\mathrm{b}) \mathrm{w}=10 \mu \mathrm{m},(\mathrm{c})$ $\mathrm{w}=5 \mu \mathrm{m},(\mathrm{d}) \mathrm{w}=1 \mu \mathrm{m}$; all figures have the same color scale.

FB waves exponentially decay in the output region which is the reason why the intensity profiles in Fig. 5(b) become broader and flat as the distance from the interface increases.

Lastly, we want to mention about the effect of the slit width on the focusing. We use the same hybridlens except now the slit width is varied from $w=1 \mu \mathrm{m}$ to $\mathrm{w}=20 \mu \mathrm{m}$. The intensity distributions of four cases are shown in Figs. 7(a)-(d) with the same color scale.

According to Fig. 7, the slit width $\mathrm{w}=20 \mu \mathrm{m}$ gives the strongest intensity while the slit width $\mathrm{w}=1 \mu \mathrm{m}$ gives the poorest intensity as shown in Figs. 7(a) and 7(d), respectively. The focal spot sizes of all cases are the same. This means that the objects whose separation distance is not less than $20 \mu \mathrm{m}$ can be resolved by all hybrid-lens but with the highest sensitivity using the slit width $\mathrm{w}=20 \mu \mathrm{m}$ and the worst sensitivity using the slit width $\mathrm{w}=1$.

The FDTD simulation is very time consuming and not accurate if the sizes of the hybrid-lens is very small comparing to the wavelength of the incident light. Therefore, the analytical theory of the hybrid-lens will give the answer to the smallest focal spot sizes that we can achieve by using the hybrid-lens. In order to fully understand this hybrid-lens analytically, one needs to combine the couple-mode theory of the multiple slits [15] with the analytical theory of the rod array as explained in the method section of this paper. Then, one needs to take into account the coupling between the slit's waveguide modes and the FB waves inside the rod array. The analytical theory is needed for the true understanding of the focusing mechanism and for further reduction of the focal spot sizes by optimizing the parameters of the hybrid-lens such as the lattice constants of the rod array, the rod's radius, the host medium, etc.

Moreover, the hybrid-lens can serve as the substrate and the subwavelength focusing lens simultaneously, and then the image of the subwavelength objects may be obtained by scanning the multiple slits. This approach is not invasive unlike the conventional near field scanning microscope which scan the apertures or the metallic tips over the samples [23]. By using the hybrid-lens, we have achieved the focal spot size about 7 times smaller than that from the fibre hyperlens because our hybrid-lens can function in higher frequency region relying on the coupling of the electromagnetic modes of the multiple slits and those of the rod array while the fibre hyperlens neglected this coupling effect and relied on the Faby-Perot resonance along the finite fibre [10]. However, we neglect the loss of the host Zeonex polymer in the FDTD simulation because the FDTD doesn't allow the definition of its single complex dielectric constant. We expect that the transmittance and the intensity contrast between the focal spot and the background will decrease and more investigation using another techniques in frequency domain such as the finite element (FE) and the analytical solutions (ANS) using eigenmode expansion are needed. We suggest to use Si instead of Zeonex polymer as the host medium because of its lower loss in the THz frequency range, $\operatorname{Im}(\varepsilon) \approx 4 \times 10^{-4}$ at $1 \mathrm{THz}$. [24]. However, the large refractive index of Si requires higher resolutions if ones use FDTD or FE and therefore the search for the ANS, which is consistent with FDTD or FE, is important to address the loss issue more efficiently. 


\section{Conclusion}

We have proposed the planar hybrid-lens consisting of the multiple slits and the rod array. The analytical theory is developed to explain the strongly anisotropic property of the rod array whose rods are arranged in the rectangular lattice. The subwavelength focal spot size about $20 \mu \mathrm{m}(\lambda / 7.5)$ is demonstrated at the frequency $2 \mathrm{THz}$ by FDTD simulation. The sensitivity of the hybrid-lens depends on the slit width. Our proposed prototype may be used to enhance the resolution of the image in the fast terahertz medical imaging.

\section{Acknowledgements}

The first author is grateful to the Institute for the Promotion of Teaching Science and Technology (IPST), Thailand, for the fully financial support in this research. The first author also thanks to the Terahertz Sensing and Imaging Laboratory in Sendai, Japan, for providing very relaxing facility for this research.

\section{References}

[1] M. Tonouchi, "Cutting-edge terahertz technology," Nat. Photon., vol. 1, pp. 97-105, Feb., 2007.

[2] S. Jae Oh, S.-H. Kim, Y. B. Ji, K. Jeong, Y. Park, J. Yang, D. W. Park, S. K. Noh, S.-G. Kang, Y.-M. Huh, J.-H. Son, and J.-S. Sue, "Study of freshy excised brain tissues using terahertz imaging," Biomed. Opt. Express, vol. 5, no. 8, pp. 2837-2842, Aug., 2014.

[3] T. Ouchi, K. Kajiki, T. Koizumi, T. Itsuji, Y. Koyama, R. Sekiguchi, O. Kubota, and K. Kawase, "Terahertz imaging system for medical applications and related high efficiency terahertz devices," $J$ Infrared Milli Terahz Waves, vol. 35, pp. 118-130, July, 2013.

[4] J. B. Pendry, "Negative refraction makes a perfect lens," Phys. Rev. Lett., vol. 85, no. 18, pp. 3966-3969, Oct., 2000.

[5] Z. Jacob, L. V. Alekseyev, and E. Narimanov, "Optical hyperlens: far-field imaging beyond the diffraction limit," Opt. Express, vol. 14, no. 18, pp. 8247-8256, Sept., 2006.

[6] Z. Liu, H. Lee, Y. Xiong, C. Sun, and X. Zhang, "Far-field optical hyperlens magnifying subdiffraction-limited objects," Science, vol. 315, no. 5819, p. 1686, Mar., 2007.

[7] P. Tapsanit, M. Yamashita, and C. Otani, "Analytical solutions of electromagnetic waves in focusing and magnifying cylindrical hyperlenses: Green's function approach," Opt. Express, vol. 22, no. 1, pp. 229-238, Jan., 2014.

[8] A. Poddubny, I. Iorsh, P. Belov, and Y. Kivshar, "Hyperbolic metamaterials," Nat. Photon., vol. 7, pp. 958-967, Dec., 2013.

[9] S. Ishii, A. V. Kildishev, E. Narimanov, V. M. Shalev, and V. P. Drachev, "Sub-wavelength interference pattern from volume plasmon polaritons in a hyperbolic medium," Laser Photon. Rev., vol. 7, no. 2, pp. 265-271, Jan., 2013.

[10] A. Tuniz, K. J. Kaltenecker, B. M. Fischer, M. Walther, S. C. Fleming, A. Argyros, and B. T. Kuhlmey, "Metamaterial fibres for subdiffraction imaging and focusing at terahertz frequencies over optically long distances," Nat. Commun., vol. 4, no. 2706, Oct., 2013.

[11] A. Tuniz, D. Ireland, L. Poladian, A. Argyros, C. M. de Sterke, and B. T. Kuhlmey, "Imaging performance of finite uniaxial metamaterials with large anisotropy," Opt. Lett., vol. 39, no. 11, pp. 3286-3289, June, 2014.

[12] L. A. Ibbotson and J. J. Baumberg, "Fabricating large-area metallic woodpile photonic crystals using stacking and rolling," Nanotechnology, vol. 24, p. 305301, June, 2013.

[13] M. A. Ordal, R. J. Bell, R. W. Alexander, Jr, L. L. Long, and M. R. Querry, "Optical properties of fourteen metals in the infrared and far infrared: $\mathrm{Al}, \mathrm{Co}, \mathrm{Cu}, \mathrm{Au}, \mathrm{Fe}, \mathrm{Pb}, \mathrm{Mo}, \mathrm{Ni}, \mathrm{Pd}, \mathrm{Pt}, \mathrm{Ag}, \mathrm{Ti}, \mathrm{V}$, and W," Appl. Opt. , vol. 24, no. 24, pp. 4493-4499, Apr., 1985.

[14] J. Anthony, R. Leonhardt, A. Argyros, and M. C. J. Large, "Characterization of a microstructured Zeonex terahertz fiber," J. Opt. Soc. Am. B , vol. 28, no. 5, pp. 1013-1018, May, 2011.

[15] A. I. Fernández-Domínguez, F. J. García-Vidal, and L. Martín-Moreno, "Resonant transmission of light through finite arrays of slits," Phys. Rev. B, vol. 76, p. 235430, Dec., 2007. 
[16] A. F. Oskooi, D. Roundy, M. Ibanescu, P. Bermel, J. D. Joannopoulos, and S. G. Johnson, "MEEP: A flexible free-software package for electromagnetic simulations by the FDTD methods," Comput. Phys. Commun., vol. 181, no. 3, pp. 687-702, Jan., 2001.

[17] G. D'Aguanno and N. Mattiucci, "Dispersive and scattering properties of multilayer arrays made of plasmonic nanoparticles," J. Opt. Soc. Am. B, vol. 31, no. 10, pp. 2524-2530, Oct., 2014.

[18] L. Novotny and B. Hecht, "Theoretical foundations," in Principle of Nano-optics, 2nd ed. Cambridge, United Kingdom: Cambridge University Press, 2012, ch. 2, sec. 2.10, pp. 20-22.

[19] F. J. García de Abajo, "Light scattering by particle and hole array," Rev. Mod. Phys., vol. 79, no. 4, pp. 1267-1290, Oct., 2007.

[20] R. Gómez-Medina, M. Laroche, and J. José Sáenz, "Extraordinary optical reflection from subwavelength cylindrical arrays," Opt. Express, vol. 14, no. 9, pp. 3730-3737, Apr., 2006.

[21] E. Kallos, I. Chremmos, and V. Yannopapas, "Resonance properties of optical all-dielectric metamaterials using two-dimensional multipole expansion," Phys. Rev. B, vol. 86, pp. 245108(10) Dec., 2012.

[22] R. J. P. Engelen, D. Mori, T. Baba, and L. Kuipers, "Subwavelength structure of the evanescent field of an optical Bloch wave," Phys. Rev. Lett., vol. 102, no. 2, pp. $023902(4)$, Jan., 2009.

[23] S. Hunsche, M. Koch, I. Brener, and M. C. Nuss, "THz near-field imaging," Opt. Commun., vol. 150, pp. 22-26, May, 1998.

[24] D. Grischkowsky, S. Keiding, M. V. Exter, and Ch. Fattinger, "Far-infrared time-domain spectroscopy with terahertz beams of dielectrics and semiconductors," J. Opt. Soc. Am. B, vol. 7, no. 10, pp. $2006-$ 2015, October, 1990. 